\title{
Dose-volume analysis of predictors for acute anal toxicity after radiotherapy in prostate cancer patients
}

\author{
Xingsi Peng ${ }^{1,2 \dagger}$, Sha Zhou ${ }^{1 \dagger}$, Shiliang Liu ${ }^{1 \dagger}$, Jibin Li ${ }^{3}$, Sijuan Huang ${ }^{1}$, Xiaobo Jiang ${ }^{1}$, Maosheng Lin', \\ Shaomin Huang ${ }^{1}$, Chengguang Lin ${ }^{1}$, Chaonan Qian², Mengzhong Liu ${ }^{1 *}$ and Liru He ${ }^{1 *}$
}

\begin{abstract}
Background: This study aimed to evaluate the clinical and dosimetric factors predictive of acute anal toxicity (AAT) after radiotherapy in prostate cancer ( $\mathrm{PCa}$ ) patients with or without hemorrhoids.

Methods: We analyzed data from 347 PCa patients (248 cases treated from July 2013 to November 2017 for training cohort and 99 cases treated in 2018 for validation cohort) treated with pelvic radiotherapy at a single institution. Anal canal dose-volume histogram was used to determine the prescribed dose. Univariate and multivariate analyses were used to evaluate the risk of AAT as a function of clinical and dosimetric factors.

Results: Totally, 39.5\% (98/248) and 31.3\% (31/99) of the PCa patients developed AAT in training and validation cohorts, respectively. The incidence of AAT was much higher in patients with hemorrhoids than in those without hemorrhoids in both training and validation cohorts. Hemorrhoids and volume received more than 20 Gy (V20) were valuated as independent factors for predicting AAT in training cohort. Similar results were also observed in our validation cohort. The combination of hemorrhoids and high anal canal V20 (> 74.93\% as determined by ROC curves) showed the highest specificity and positive predictive values for predicting AAT in both training and validation cohorts.
\end{abstract}

Conclusions: AAT occurs commonly in PCa patients with hemorrhoids during and after pelvic radiotherapy. Hemorrhoids and anal canal V20 are independent predictors of AAT. These factors should be carefully considered during treatment planning to minimize the incidence of AAT.

Keywords: Prostate cancer, Radiotherapy, Hemorrhoid, Acute anal toxicity

\section{Introduction}

Prostate cancer $(\mathrm{PCa})$ is the most frequently diagnosed cancer among men in America and Europe, and its incidence is rapidly increasing in Asian counties, including China [1-4]. With the increasing use of radiotherapy (RT) in the curative management of this disease over the last few decades, the potential radiotoxicity has drawn increased attention.

\footnotetext{
* Correspondence: liumzh@sysucc.org.cn; helir@sysucc.org.cn Xingsi Peng and Jibin Li were responsible for statistical analyses 'Department of Radiation Oncology, Sun Yat-Sen University Cancer Center, State Key Laboratory of Oncology in South China, Collaborative Innovation Center for Cancer Medicine, Guangzhou, China

Full list of author information is available at the end of the article
}

With respect to gastrointestinal toxicity, a number of reports have described rectal [5-7] or anorectal toxicity [8-10], which manifests in symptoms such as acute diarrhea, fecal incontinence, chronic proctitis, and rectal bleeding. Symptoms of acute anal toxicity (AAT), especially anal pain or bleeding, tend to be ignored because of their relatively lower severities. However, anal pain and bleeding are quite common symptoms during pelvic radiotherapy for Asian patients. Two studies from Korea indicated that hemorrhoids are an important risk factor for AAT in patients undergoing pelvic radiotherapy [11, 12]. However, both studies only enrolled small numbers of patients (31 and 33 patients, respectively) and included cases with different cancers and different radiation 
techniques, which made these data inadequate for reliable dosimetric analysis.

The prevalence of hemorrhoids is reported to be up to $90 \%$ in China [13], which is much higher than that in Western Europe and the United States [14, 15]. Chinese patients with PCa show a much higher risk of AAT after radiotherapy. However, we still have no clear idea about how to help the hemorrhoids patients to reduce the risk of AAT. And the dosimetric recommendations of anal canal for preventing AAT have long been lacking. The purpose of our study was to determine the risk factors, in particular the dosimetric factors, for AAT in Chinese PCa patients who were treated with radiotherapy at our institution.

\section{Methods}

\section{Patient selection}

The institutional review board approved this retrospective study and waived the need to obtain informed consent. We retrospectively reviewed $248 \mathrm{PCa}$ patients treated at the Radiation Oncology Department of our institution between July 2013 and November 2017. As a validation, 99 PCa patients treated with pelvic radiotherapy in 2018 at the same institution were also studied. The following criteria were used to select the patients for our study: pathologically confirmed diagnosis of prostatic adenocarcinoma; definitive radiotherapy to the primary tumor or postoperative radiotherapy to the tumor bed; the availability of anal dose-volume histograms (DVHs) in our institutional archives; and the availability of weekly patient records during the treatment and post-radiotherapy clinical assessments of patients' AAT. Exclusion criteria were previous pelvic radiotherapy, secondary malignancy, and unknown critical clinical information.

\section{Simulation and contouring}

Simulation CT was performed with 3-mm slices in the prone position on a belly board. All the patients had made dietary preparation at least 1 week prior to the day of the $\mathrm{CT}$ scan. And cone beam CT or portal imaging was performed in general for the patients. The CT image was transferred to the workstation (Monaco V5.11, Elekta AB, Stockholm, Sweden), and the target volumes and critical organs were contoured. Clinical target volumes were contoured according to the recommendations of the Radiation Therapy Oncology Group (RTOG). Clinical target volumes were expanded by $5-7 \mathrm{~mm}(3-5 \mathrm{~mm}$ posterior) to produce planning target volumes (PTVs). The location of the anal verge was defined on the basis of the last caudal image of the external sphincter muscle in CT images. The anal canal volume was defined as the volume from the anal verge to 3 $\mathrm{cm}$ superior to the anal verge in planning CT images.

\section{Treatment approaches}

Patients with intermediate, high, or very high-risk $\mathrm{PCa}$ and patients underwent salvage radiotherapy postoperatively received androgen deprivation therapy before, during, and after radiotherapy. Patients with low-risk $\mathrm{PCa}$ or those treated postoperatively with an adjuvant intent received radiotherapy alone. Patients with low risk did not receive pelvic RT. Postoperative patients with regional lymph nodes metastasis received pelvic RT. The radiation doses for the pelvic were $45 \sim 50 G y / 25 \mathrm{~F}$. The conventional fraction modality (1.8-2.0 Gy per fraction) was usually used to treat PCa patients postoperatively, whereas the hypofractioned modality (2.4-3.0 Gy per fraction) was only prescribed to $\mathrm{PCa}$ patients with definitive treatment intent. Before October 2014, patients received intensity-modulated radiotherapy (IMRT) or volumetric-modulated arc therapy (VMAT), and after October 2014, all the patients were treated by imageguided IMRT or VMAT.

\section{Clinical evaluation and follow-up}

During radiotherapy, symptoms were closely monitored on a weekly basis and even more frequently if required for clinical evaluation and disease management. After completing radiotherapy, all patients were followed up every 3 months for the first 2 years and every 6-12 months thereafter. The median follow-up time is 36 months. And all patients were followed up more than 3 months. As we observed in clinical assessments, the main symptoms of AAT were anal pain and anal bleeding. We graded AATs according to the National Cancer Institute-Common Terminology Criteria for Adverse Events version 4.03 (NCI-CTCv4.03) as follows: Grade 1: mild bleeding without intervention indicated and/or mild pain not interfering with function; Grade 2: symptomatic bleeding requiring medical intervention or minor cauterization, and/or moderate pain interfering with function, but not interfering with activities of daily living (ADLs); Grade 3: bleeding that required transfusion, interventional radiology, endoscopic, or operative intervention, and/or severe pain severely interfering with ADL; Grade 4: life-threatening bleeding consequences with major urgent intervention indicated and/or disabling pain; Grade 5: Death.

\section{Statistical analysis}

Descriptive statistics (e.g., means, standard deviation, and percentages) were presented when appropriate. The differences in the clinical and DVH characteristics between the AAT and NAAT groups were compared using Mann-Whitney's $U$ test for quantitative variables and Fisher's exact test for categorical variables. Multivariate logistic regression models were created to evaluate the risk of AAT as a function of clinical and dosimetric 
factors. The factors with $p<0.05$ in univariate analyses were involved in subsequent multivariate analyses with forward stepwise analyses to assess the independent factors associated with the risk of AAT. Receiver operating characteristic (ROC) curve analyses were performed for DVH parameters to select the most relevant threshold to differentiate symptomatic AAT. The optimal threshold for each DVH parameter was defined as the point yielding the minimal value for $(1 \text { - sensitivity })^{2}+(1$ - specificity $)^{2}$, which was the point on the ROC curve closest to the upper left-hand corner $(0,1)[16]$. A $P$ value $<0.05$ was considered statistically significant. All analyses were performed using SPSS (version 20.0, Chicago).

\section{Results}

\section{Patient characteristics}

The characteristics of the 248 patients from training cohort and 99 patients from validation cohort are summarized in Table 1. The median age of the two group patient cohorts was 67 years. One hundred and thirtynine patients $(56.0 \%)$ and 52 patients (52.5\%) reported hemorrhoids before radiotherapy in training and validation cohorts, respectively. The majority $(71.76 \%)$ of the patients received androgen deprivation therapy before, during, and after RT. The radiation dose per fraction ranged from $1.8 \mathrm{~Gy}$ and $3.0 \mathrm{~Gy}$, and the total dose varied from 60 Gy to $81 \mathrm{~Gy}$. The majority of the patients (303/ $347,87.3 \%)$ received image-guided radiotherapy (IGRT), and the other patients received IMRT or VMAT. Portal imaging was performed before the initiation of the IMRT/VMAT course, while cone beam CT was performed before each treatment for patients treated with IGRT.

\section{Clinical characteristics and acute anal toxicity}

Totally, 39.5\% (98/248) and 31.3\% (31/99) of the PCa patients developed AAT in training and validation cohorts, respectively. There were 89 (35.8\%), 8 (3.2\%) and $1(0.4 \%)$ patients developed grade 1 , grade 2 and grade 3 AAT in training cohort. While 29 (29.3\%), $2(2.0 \%)$ and $0(0.0 \%)$ of the patients reported grade 1 , grade 2 and grade 3 AAT in validation cohort. No grade 4 or grade 5 AATs were observed in both cohorts. At the beginning of radiotherapy, the majority of the patients were asymptomatic, and only 15 patients reported mild anal pain and/or bleeding. All 15 symptomatic patients complained of anal symptom aggravation during the course of radiotherapy, including 3 patients (Grade 2 and 3 anal bleeding for 2 and 1 patients, respectively) who had to suspend radiotherapy for more than 2 weeks to receive medical intervention. The median time to the initiation of anal symptoms was 3 weeks (range, 0 to 5 weeks), with a corresponding median induction dose of about 37.5 Gy (EQD2:38.3Gy, $\alpha / \beta$ for anal canal 4).
In both cohorts, hemorrhoids were evaluated as the only clinical factor that showed a significant association with AAT (Table 1). The incidence of AAT was much higher in patients with hemorrhoids than in those without hemorrhoids in training cohort $(60.4 \%$ vs. $12.8 \%$, $P<0.001)$ and validation cohort $(44.2 \%$ vs. $17.0 \%$, $P=0.004)$. No significant differences were found between the no-acute anal toxicity (NAAT) groups and AAT groups with respect to the other clinical variables, such as age, $\mathrm{T}$ stage, $\mathrm{N}$ stage, $\mathrm{M}$ stage, clinical stage, GS group, TPSA before treatment or before RT, RT type, RT modality, Surgery and Hormonal treatment duration in both training and validation cohorts (Table 1).

\section{Dose-volume parameters and acute anal toxicity}

In both training and validation cohorts, the mean prescribed dose of radiotherapy and the mean volume of the anal canal were not significantly different between the NAAT and AAT groups (Table 2). The maximum dose for the anal canal for the AAT group was significantly higher than those for the NAAT group in training cohort, while it was not significantly in validation cohort $(P=0.018$ and $P=0.169$, Table 2$)$. In addition, in both cohorts, AAT patients showed significantly higher mean dose for anal canal, V10, V20, V30, V40, V50 and V60 values than NAAT patients $(P<0.05$, Table 2$)$.

\section{Multivariate regression analysis of acute anal toxicity}

Hemorrhoids and the dose-volume parameters factors that showed significance in univariate analysis were further analyzed in the multivariate regression model (Table 3). In both training and validation cohorts, only hemorrhoids $(\mathrm{OR}=10.94$ and 5.34, respectively, $P<0.001)$ and V20 (OR $=1.03$ and 1.04, respectively, $P<0.001)$ were found to be independent predictors of AAT.

\section{The anal canal V20 and the risk of acute anal toxicity}

To confirm the effect of the anal canal V20 on the risk of AAT, we grouped the patients from the training cohort into ten equal-sized bins in terms of patient number and plotted the incidence risk vs. the V20. The solid curve in Fig. 1 shows the fit of the logistic model to the data and the trend toward an increased risk of AAT with a higher anal canal V20. There is an average increase of $2.1 \%$ in AAT probability for each incremental $1 \%$ rise in the anal canal V20.

ROC curve analysis was performed in the training cohort to select the most relevant dose-volume parameter to predict AAT. The optimal threshold of the anal canal V20, with an area under the ROC curve (AUC) of 0.653 (sensitivity: 0.541, specificity: 0.700, Fig. 2). The incidence of AAT was significantly higher in patients with an anal canal V20 more than $74.93 \%$ than in those 
Table 1 Patient characteristics

\begin{tabular}{|c|c|c|c|c|c|c|}
\hline \multirow{2}{*}{ Factors } & \multicolumn{3}{|c|}{ Training group ( $N=248$ cases) } & \multicolumn{3}{|c|}{ Validation group ( $N=99$ cases) } \\
\hline & NAAT & AAT & $P$ & NAAT & AAT & $P$ \\
\hline \multicolumn{7}{|l|}{ Age (\%) } \\
\hline $46-68 y s$ & $72(55.8)$ & $57(44.2)$ & 0.117 & $44(75.9)$ & $14(24.1)$ & 0.067 \\
\hline $68-88 y s$ & $78(65.5)$ & $41(34.5)$ & & $24(58.5)$ & $17(41.5)$ & \\
\hline \multicolumn{7}{|l|}{ T stage (\%) } \\
\hline $1 \sim 2$ & $36(56.3)$ & $28(43.7)$ & 0.789 & $21(67.7)$ & $10(32.3)$ & 0.933 \\
\hline $3 \sim 4$ & $114(62.0)$ & $70(38.0)$ & & $45(69.2)$ & $20(30.8)$ & \\
\hline \multicolumn{7}{|l|}{ N stage (\%) } \\
\hline 0 & $92(64.3)$ & $51(35.7)$ & 0.148 & $40(65.6)$ & $21(34.4)$ & 0.375 \\
\hline 1 & $58(55.2)$ & $47(44.8)$ & & $26(74.3)$ & $9(25.7)$ & \\
\hline \multicolumn{7}{|l|}{ M stage (\%) } \\
\hline 0 & $90(60.4)$ & $59(39.6)$ & 0.974 & $48(69.6)$ & $21(30.4)$ & 0.775 \\
\hline 1 & $60(60.6)$ & $39(39.4)$ & & $20(66.7)$ & $10(33.3)$ & \\
\hline \multicolumn{7}{|l|}{ Clinical stage (\%) } \\
\hline $\mid \sim \|$ & $23(54.8)$ & $19(45.2)$ & 0.753 & $18(66.7)$ & $9(33.3)$ & 0.880 \\
\hline$\| I|\sim| V$ & $127(61.7)$ & 79 (38.3) & & $50(69.4)$ & $22(30.6)$ & \\
\hline \multicolumn{7}{|l|}{ GS group (\%) } \\
\hline $6 \sim 7$ & $66(64.7)$ & $36(35.3)$ & 0.256 & $25(80.6)$ & $6(19.4)$ & 0.074 \\
\hline $8 \sim 10$ & $84(57.5)$ & $62(42.5)$ & & $40(62.5)$ & $24(37.5)$ & \\
\hline \multicolumn{7}{|l|}{ TPSA before treatment (\%) } \\
\hline$<20 \mathrm{ng} / \mathrm{mL}$ & $22(59.5)$ & $15(40.5)$ & 0.890 & $40(62.5)$ & $24(37.5)$ & 0.226 \\
\hline$\geq 20 \mathrm{ng} / \mathrm{mL}$ & $128(60.7)$ & $83(39.3)$ & & $19(76.0)$ & $6(24.0)$ & \\
\hline \multicolumn{7}{|l|}{ TPSA before RT (\%). } \\
\hline$<20 \mathrm{ng} / \mathrm{mL}$ & $119(60.1)$ & 79 (39.9) & 0.806 & $61(67.0)$ & $30(33.0)$ & 0.407 \\
\hline$\geq 20 \mathrm{ng} / \mathrm{mL}$ & $31(62.0)$ & $19(28.0)$ & & $5(83.3)$ & $1(16.7)$ & \\
\hline \multicolumn{7}{|l|}{ RT type (\%) } \\
\hline IMRTNMMAT & $23(67.6)$ & $11(32.4)$ & 0.358 & $5(50.0)$ & $5(50.0)$ & 0.179 \\
\hline IMRTNMAT-IGRT & $127(59.3)$ & $87(40.7)$ & & $63(70.8)$ & $26(29.2)$ & \\
\hline \multicolumn{7}{|l|}{ RT modality (\%) } \\
\hline Conventional fraction & $126(62.1)$ & 77 (37.9) & 0.278 & $46(66.7)$ & $23(33.3)$ & 0.511 \\
\hline Hypo-fraction & $24(53.3)$ & $21(46.7)$ & & $22(73.3)$ & $8(26.7)$ & \\
\hline \multicolumn{7}{|l|}{ Surgery (\%) } \\
\hline No & $94(61.4)$ & 59 (38.6) & 0.697 & $31(62.0)$ & $19(38.0)$ & 0.483 \\
\hline Yes & $56(58.9)$ & $39(41.1)$ & & $33(68.8)$ & $15(31.3)$ & \\
\hline \multicolumn{7}{|c|}{ Hormonal treatment duration (\%) } \\
\hline$<2$ years & $23(54.8)$ & $19(45.2)$ & 0.405 & $17(63.0)$ & $10(37.0)$ & 0.730 \\
\hline$\geq 2$ years & $127(61.7)$ & $79(38.3)$ & & $48(66.7)$ & $24(33.3)$ & \\
\hline \multicolumn{7}{|l|}{ Hemorrhoid (\%) } \\
\hline NO & $95(87.2)$ & $14(12.8)$ & $<0.001$ & $39(83.0)$ & $8(17.0)$ & 0.004 \\
\hline Yes & 55 (39.6) & $84(60.4)$ & & $29(55.8)$ & $23(44.2)$ & \\
\hline
\end{tabular}


Table 2 Univariate analysis of DVH parameters related to AAT

\begin{tabular}{|c|c|c|c|c|c|c|}
\hline \multirow[b]{2}{*}{ Variables } & \multicolumn{3}{|c|}{ Training group ( $N=248$ cases) } & \multicolumn{3}{|c|}{ Validation group ( $N=99$ cases) } \\
\hline & NAAT $($ Mean \pm SD) & AAT (Mean \pm SD) & P & NAAT (Mean \pm SD) & AAT (Mean \pm SD) & $P$ \\
\hline Prescribed Dose (Gy) & $67.04 \pm 5.02$ & $67.33 \pm 5.77$ & 0.672 & $69.12 \pm 4.65$ & $67.35 \pm 3.96$ & 0.073 \\
\hline Volume of anal canal $\left(\mathrm{cm}^{3}\right)$ & $9.43 \pm 4.02$ & $10.02 \pm 3.60$ & 0.232 & $9.33 \pm 3.51$ & $8.00 \pm 3.17$ & 0.080 \\
\hline Anal canal Dmax (Gy) & $59.51 \pm 12.95$ & $63.22 \pm 9.65$ & 0.018 & $54.47 \pm 13.83$ & $58.40 \pm 11.13$ & 0.169 \\
\hline Anal canal Dmean (Gy) & $26.04 \pm 12.60$ & $30.25 \pm 9.71$ & 0.010 & $22.51 \pm 9.61$ & $29.43 \pm 11.52$ & 0.005 \\
\hline V10 (\%) & $75.61 \pm 22.45$ & $84.48 \pm 18.89$ & 0.002 & $67.82 \pm 22.35$ & $85.52 \pm 18.06$ & 0.001 \\
\hline V20 (\%) & $58.41 \pm 26.62$ & $72.62 \pm 25.59$ & $<0.001$ & $49.71 \pm 29.09$ & $74.24 \pm 23.84$ & $<0.001$ \\
\hline V30 (\%) & $37.81 \pm 24.32$ & $52.08 \pm 26.91$ & $<0.001$ & $30.65 \pm 27.74$ & $49.85 \pm 30.98$ & 0.006 \\
\hline V40 (\%) & $19.31 \pm 16.18$ & $27.30 \pm 20.44$ & 0.001 & $13.75 \pm 14.98$ & $27.46 \pm 25.60$ & 0.009 \\
\hline V50 (\%) & $8.81 \pm 9.09$ & $13.40 \pm 13.01$ & 0.002 & $5.22 \pm 6.40$ & $12.83 \pm 15.38$ & 0.011 \\
\hline V60 (\%) & $3.16 \pm 4.33$ & $5.34 \pm 7.28$ & 0.006 & $1.66 \pm 2.83$ & $3.95 \pm 5.59$ & 0.038 \\
\hline V70 (\%) & $0.08 \pm 0.31$ & $0.44 \pm 2.13$ & 0.099 & $0.07 \pm 0.24$ & $0.05 \pm 0.27$ & 0.785 \\
\hline
\end{tabular}

Abbreviations: DVH dose-volume histograms; AAT acute anal toxicity; NAAT no-acute anal toxicity; Dmax maximum dose; Dmean mean dose; V10-V70 percentage of anal canal volume receiving more than $10-70 \mathrm{~Gy}$

with an anal canal V20 less than 74.93\% (54.08\% vs. $30.00 \%, P<0.001)$.

\section{Predictive model analysis of acute anal toxicity}

To identify the most optimal predictive model for AATs, we further analyzed the sensitivity, specificity, positive predictive value $(+\mathrm{PV})$, negative predictive value $(-\mathrm{PV})$, and AUC of hemorrhoids and anal canal V20 in both training cohort and validation cohort. As shown in Table 4 , hemorrhoids showed the best sensitivity ( 85.71 and $74.19 \%)$ and -PV (87.16 and $82.98 \%)$ in predicting AAT, while the combination of hemorrhoids and anal canal V20 > 74.93\% showed the highest specificity $(91.33$ and $94.12 \%$ ) and + PV (77.97 and 75.00\%) for predicting AAT in training and validation cohorts, respectively.

\section{Discussion}

One of the important findings in this study is that hemorrhoids are the most important risk factor for predicting AAT. After the beginning of pelvic irradiation,

Table 3 Multivariate regression analysis related to AAT

\begin{tabular}{|c|c|c|c|}
\hline Groups & Variables & OR $(95 \% \mathrm{Cl})$ & $P$ \\
\hline \multirow[t]{9}{*}{ Training group ( $N=248$ cases) } & V10 (\%) & $1.02(1.01 \sim 1.03)$ & 0.650 \\
\hline & V20 (\%) & $1.03(1.01 \sim 1.04)$ & $<0.001$ \\
\hline & V30 (\%) & $1.02(1.01 \sim 1.03)$ & 0.607 \\
\hline & V40 (\%) & $1.02(1.01 \sim 1.04)$ & 0.947 \\
\hline & V50 (\%) & $1.04(1.01 \sim 1.07)$ & 0.847 \\
\hline & V60 (\%) & $1.07(1.02 \sim 1.13)$ & 0.526 \\
\hline & Anal canal Dmax (Gy) & $1.00(0.96 \sim 1.04)$ & 0.994 \\
\hline & Anal canal Dmean (Gy) & $1.00(0.96 \sim 1.05)$ & 0.810 \\
\hline & Hemorrhoid (cases) & $10.94(5.47 \sim 21.89)$ & $<0.001$ \\
\hline \multirow[t]{8}{*}{ Validation group ( $N=99$ cases) } & V10 (\%) & $1.04(1.02 \sim 1.06)$ & 0.478 \\
\hline & V20 (\%) & $1.04(1.02 \sim 1.06)$ & $<0.001$ \\
\hline & V30 (\%) & $1.02(1.01 \sim 1.04)$ & 0.461 \\
\hline & V40 (\%) & $1.03(1.01 \sim 1.06)$ & 0.676 \\
\hline & V50 (\%) & $1.06(1.01 \sim 1.11)$ & 0.438 \\
\hline & V60 (\%) & $1.12(1.01 \sim 1.24)$ & 0.559 \\
\hline & Anal canal Dmean (Gy) & $1.06(1.02 \sim 1.11)$ & 0.712 \\
\hline & Hemorrhoid (cases) & $5.34(1.85 \sim 15.39)$ & $<0.001$ \\
\hline
\end{tabular}




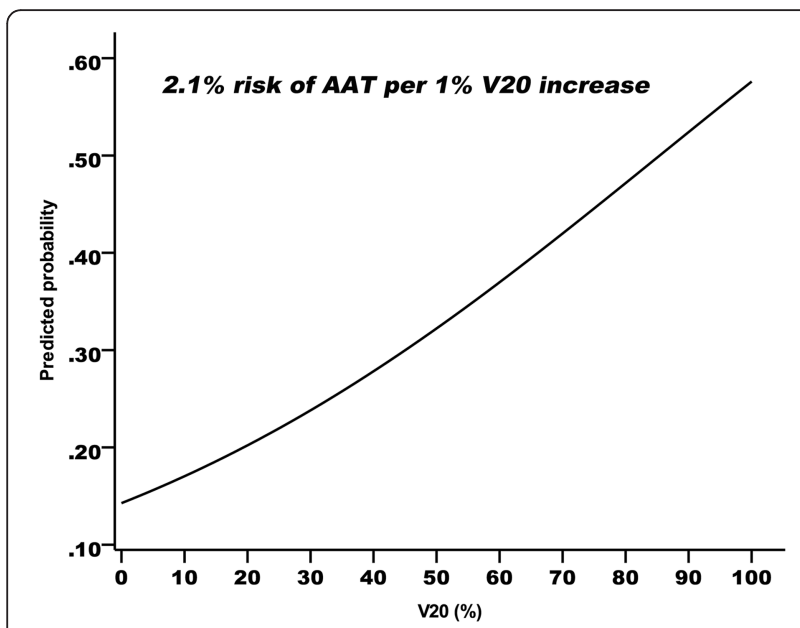

Fig. 1 The predicted probability of radiation-related anal reaction as a function of the relative anal volume treated with 20 Gy radiation (V20) by using logistic regression model

anal symptoms developed or worsened in $84(60.4 \%)$ of 139 patients with hemorrhoids and only in $14(12.8 \%)$ of 109 patients without hemorrhoids. Similar results were also observed in validation cohort, in which AAT developed in $44.2 \%$ of the patients with hemorrhoids, but only in $17.0 \%$ of the patients without hemorrhoids. Our results also confirm the high frequency of AAT (42.4-50\%) after whole pelvic irradiation of $45-50.4 \mathrm{~Gy}$ for pelvic malignant disease reported previously in Korean patients with asymptomatic hemorrhoids [11, 12]. Interestingly, few studies from America and Europe have addressed this

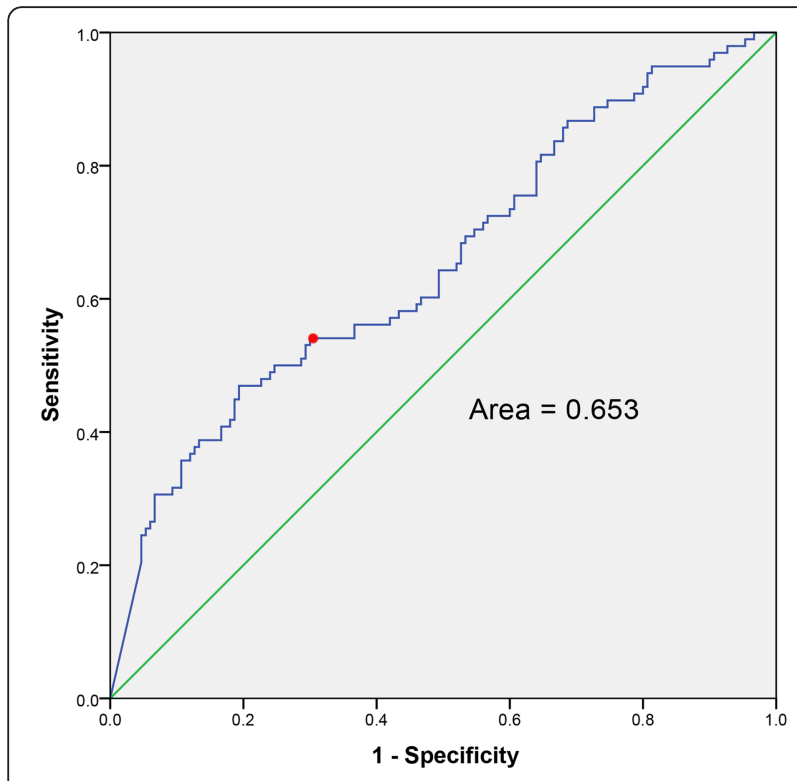

Fig. 2 ROC curves of all patients and the associated area for V20 as a predictor for AAT. The optimal threshold value is $74.93 \%$ (plotted using red circle) and corresponds to a sensitivity of 0.541 and specificity of 0.700 topic, mainly because both hemorrhoids and AAT are less common in patients in these regions, and the symptoms are usually mild and resolve spontaneously [5, 17, 18]. However, we observed three patients with symptomatic hemorrhoids at the beginning of radiotherapy show severe bleeding that necessitated suspension of radiotherapy for more than 2 weeks to allow medical intervention. On the contrary, in the 11 patients who had a history of surgical removal of hemorrhoids before, no one reported $\geq$ Grade 2 AAT symptoms after radiotherapy. The presence of symptomatic hemorrhoids at the beginning of radiotherapy was expected to be associated with a higher risk for symptom aggravation. Nevertheless, the findings still did not provide a clear idea about preventing AAT. Medical treatment to control anal symptoms in patients with symptomatic hemorrhoids before the initiation of radiotherapy might be one of the possible solutions to reduce the risk and the severity of AAT. However, since the symptoms of AAT are quite different from those of acute radiation-induced proctitis, which are commonly diarrhea and defecation urgency [19-21], it is necessary to distinguish the anal canal from the rectum to seek proper dosimetric recommendations in clinical practice.

Although radiation doses of 45 to $55 \mathrm{~Gy}$ in fractions of 1.8 to $2 \mathrm{~Gy}$ are considered to be safe for the anal canal [22], patients with hemorrhoids may be troubled even by low radiation doses. The median induction dose for AAT is about 37.5 Gy (38.3 Gy calculated as EQD2) in our study, which is similar to the results of the two studies from Korea (34.1-36.9 Gy) [11, 12]. However, none of the DVH parameters were significant predictors of AAT in their studies, and only V10 or V30 and V40 showed marginal correlations with AAT [11, 12]. Indeed, it is difficult to draw reliable conclusions from two studies with such small sample sizes of 31 and 33 patients, and DVH predictors of AAT should be re-evaluated in larger patient cohorts. In the present study, we enrolled $248 \mathrm{PCa}$ patients treated with pelvic radiotherapy as a training cohort, and our results showed that the maximum and mean dose as well as the V10, V20, V30, V40, V50, V60, and V70 for the anal canal were significantly higher in the AAT group. However, when all these parameters including hemorrhoids were assessed in the multivariate analysis, V20 was the only independent dosimetric factor predicting AAT. Similar results were also observed in our validation cohort.

As noted in our study, the AAT probability increased by only $2.1 \%$ for each incremental $1 \%$ increase in the anal canal V20, and hemorrhoids remained the most dominant risk factor for AAT. Furthermore, we generated models including hemorrhoids and V20 to predict AAT, and found that any V20 without hemorrhoids had a low +PV, whereas high V20 (>74.93\%) with hemorrhoids had a much higher +PV than low V20 (<74.93\%) 
Table 4 Predictive value analysis of hemorrhoids and/or anal canal V20

\begin{tabular}{|c|c|c|c|c|c|c|c|c|}
\hline Groups & Models & AAT (cases) & NAAT (cases) & Sensitivity (\%) & Specificity(\%) & $\begin{array}{l}+P V \\
\text { (\%) }\end{array}$ & $\begin{array}{l}-P V \\
(\%)\end{array}$ & AUC \\
\hline \multirow[t]{18}{*}{$\begin{array}{l}\text { Training group } \\
(N=248 \text { cases })\end{array}$} & \multicolumn{8}{|c|}{ Model 1: Anal canal V20 } \\
\hline & High(V20 > 74.93\%) & 53 & 45 & 54.01 & 70.00 & 54.08 & 70.00 & 0.620 \\
\hline & Low(V20 $\leq 74.93 \%)$ & 45 & 105 & & & & & \\
\hline & \multicolumn{8}{|c|}{ Model 2: Hemorrhoid } \\
\hline & Yes & 84 & 55 & 85.71 & 63.33 & 60.43 & 87.16 & 0.745 \\
\hline & No & 14 & 95 & & & & & \\
\hline & \multicolumn{8}{|c|}{ Model 3: } \\
\hline & High V20 with hemorrhoid & 46 & 13 & 46.94 & 91.33 & 77.97 & 72.49 & 0.691 \\
\hline & Others & 52 & 137 & & & & & \\
\hline & \multicolumn{8}{|c|}{ Model 4: } \\
\hline & High V20 without hemorrhoid & 7 & 32 & 7.14 & 78.67 & 17.95 & 56.46 & 0.429 \\
\hline & Others & 91 & 118 & & & & & \\
\hline & \multicolumn{8}{|c|}{ Model 5: } \\
\hline & Low V20 with hemorrhoid & 38 & 42 & 38.78 & 72.00 & 47.50 & 68.35 & 0.554 \\
\hline & Others & 50 & 108 & & & & & \\
\hline & \multicolumn{8}{|c|}{ Model 6: } \\
\hline & Low V20 without hemorrhoid & 7 & 63 & 7.14 & 58.00 & 10.00 & 48.88 & 0.326 \\
\hline & Others & 91 & 87 & & & & & \\
\hline \multirow[t]{18}{*}{$\begin{array}{l}\text { Validation group } \\
(N=99 \text { cases })\end{array}$} & \multicolumn{8}{|c|}{ Model 1: Anal canal V20 } \\
\hline & High(V20 > 74.93\%) & 18 & 13 & 58.06 & 80.88 & 58.06 & 80.88 & 0.665 \\
\hline & Low(V20 $\leq 74.93 \%)$ & 13 & 55 & & & & & \\
\hline & \multicolumn{8}{|c|}{ Model 2: Hemorrhoid } \\
\hline & Yes & 23 & 29 & 74.19 & 57.35 & 44.23 & 82.98 & 0.658 \\
\hline & No & 8 & 39 & & & & & \\
\hline & \multicolumn{8}{|c|}{ Model 3: } \\
\hline & High V20 with hemorrhoid & 11 & 4 & 35.48 & 94.12 & 75.00 & 76.19 & 0.631 \\
\hline & Others & 20 & 64 & & & & & \\
\hline & \multicolumn{8}{|c|}{ Model 4: } \\
\hline & High V20 without hemorrhoid & 7 & 9 & 22.58 & 86.76 & 43.75 & 71.08 & 0.534 \\
\hline & Others & 24 & 59 & & & & & \\
\hline & \multicolumn{8}{|c|}{ Model 5: } \\
\hline & Low V20 with hemorrhoid & 14 & 23 & 45.16 & 66.18 & 37.84 & 72.58 & 0.529 \\
\hline & Others & 17 & 45 & & & & & \\
\hline & \multicolumn{8}{|c|}{ Model 6: } \\
\hline & Low V20 without hemorrhoid & 2 & 29 & 6.45 & 57.35 & 6.45 & 57.35 & 0.306 \\
\hline & Others & 29 & 39 & & & & & \\
\hline
\end{tabular}


with hemorrhoids in both training and validation cohorts. These data indicated that anal canal V $20<74.93 \%$ may serve as a proper dosimetric recommendation to reduce the risk of AAT in PCa patients with hemorrhoids.

Our study has some limitations. First, detailed information regarding the types and grades of hemorrhoids before radiotherapy were not available for most patients; second, the associations between dosimetric parameters and different grades of AAT were not analyzed because of the low incidence of Grade $\geq 2$ AAT; third, all the patients of this study performed prostate radiotherapy in prone position, while patients' position may also contribute to the higher anal dose and the present of AAT. Further studies are still needed to confirm our results.

\section{Conclusions}

AAT is common among PCa patients with hemorrhoids during and after pelvic radiotherapy. Hemorrhoids and anal canal V20 are independent predictors of AAT, which should be carefully considered during treatment planning to minimize the incidence of AAT.

\begin{abstract}
Abbreviations
+PV: Positive predictive value; AAT: Acute anal toxicity; DVHs: Dose-volume histograms; IMRT: Intensity-modulated radiotherapy; NAAT: No-acute anal toxicity; PCa: Prostate cancer; PTVs: Planning target volumes; -PV: Negative predictive value; ROC: Receiver operating characteristic; RT: Radiotherapy; V20: Volume received more than $20 \mathrm{~Gy}$; VMAT: Volumetric-modulated arc therapy
\end{abstract}

\section{Acknowledgements}

Not applicable.

\section{Authors' contributions}

PXS, ZS and LSL participated in study design, collected the clinical data and drafted the manuscript. PXS and LJB conducted the statistical analysis. HSJ, JXB, LMS, HSM, LCG and QCN contributed to the operation work during treatment. LMZ and HLR conceived of the study, participated in its design and revised the manuscript. All authors read and approved the final manuscript.

\section{Funding}

Not applicable.

\section{Availability of data and materials}

The dataset used and analysed during the current study are available from the corresponding author on reasonable request.

\section{Ethics approval and consent to participate}

This project was approved by the Ethical Committee of Sun Yat-Sen University Cancer Center and informed consent was obtained from all patients.

\section{Consent for publication}

All authors gave their consent for publication.

\section{Competing interests}

The authors declare that they have no competing interests.

\section{Author details}

'Department of Radiation Oncology, Sun Yat-Sen University Cancer Center, State Key Laboratory of Oncology in South China, Collaborative Innovation Center for Cancer Medicine, Guangzhou, China. ${ }^{2}$ Department of Nasopharyngeal Carcinoma, Sun Yat-sen University Cancer Center; State Key Laboratory of Oncology in South China; Collaborative Innovation Center of
Cancer Medicine, Guangzhou, Guangdong 510060, People's Republic of China. ${ }^{3}$ Department of Clinical Research, Sun Yat-Sen University Cancer Center, State Key Laboratory of Oncology in South China, Collaborative Innovation Center for Cancer Medicine, Guangzhou, China.

Received: 4 July 2019 Accepted: 5 September 2019

Published online: 10 October 2019

\section{References}

1. Bray F, Ferlay J, Soerjomataram I, Siegel RL, Torre LA, Jemal A. Global cancer statistics 2018: GLOBOCAN estimates of incidence and mortality worldwide for 36 cancers in 185 countries. CA Cancer J Clin. 2018;68(6):394-424.

2. Zhu Y-P, Ye D-W, Yao X-D, Zhang S-L, Dai B, Zhang H-L, Shen Y-J, Zhu Y, Shi $\mathrm{G}-\mathrm{H}$. Prevalence of incidental prostate cancer in patients undergoing radical cystoprostatectomy: data from China and other Asian countries. Asian J Androl. 2008;11(1):104-8.

3. McCracken M, Olsen M, Chen MS Jr, Jemal A, Thun M, Cokkinides V, Deapen D, Ward E. Cancer incidence, mortality, and associated risk factors among Asian Americans of Chinese, Filipino, Vietnamese, Korean, and Japanese ethnicities. CA Cancer J Clin. 2007;57(4):190-205.

4. Sim HG, Cheng CW. Changing demography of prostate cancer in Asia. Eur J Cancer. 2005:41(6):834-45.

5. Linton KD, Catto JW. Gastrointestinal toxicity following radiotherapy for prostate cancer: a ring of fire. Eur Urol. 2011;60(5):917-9.

6. Kelsey R. Prostate cancer: Hypofractionation not associated with reduced toxicity. Nat Rev Urol. 2015;12(3):126.

7. Fang P, Mick R, Deville C, Both S, Bekelman JE, Christodouleas JP, Guzzo TJ, Tochner Z, Hahn SM, Vapiwala N. A case-matched study of toxicity outcomes after proton therapy and intensity-modulated radiation therapy for prostate cancer. Cancer. 2015;121(7):1118-27.

8. Krol R, McColl GM, Hopman WPM, Smeenk RJ. Anal and rectal function after intensity-modulated prostate radiotherapy with endorectal balloon. Radiother Oncol. 2018;128(2):364-8.

9. Smeenk RJ, Hoffmann AL, Hopman WP, van Lin EN, Kaanders JH. Doseeffect relationships for individual pelvic floor muscles and anorectal complaints after prostate radiotherapy. Int J Radiat Oncol Biol Phys. 2012; 83(2):636-44.

10. Yeoh EK, Russo A, Botten R, Fraser R, Roos D, Penniment M, Borg M, Sun WM. Acute effects of therapeutic irradiation for prostatic carcinoma on anorectal function. Gut. 1998;43(1):123-7.

11. Jang $\mathrm{H}$, Baek JG, Jo S. The anal canal as a risk organ in cervical cancer patients with hemorrhoids undergoing whole pelvic radiotherapy. Tumori. 2015;101(1):72-7.

12. Jang H, Baek JG, Yoo SJ. Acute anal toxicity after whole pelvic radiotherapy in patients with asymptomatic haemorrhoids: identification of dosimetric and patient factors. Br J Radiol. 2015;88(1050):20150022.

13. Su MY, Chiu CT, Lin WP, Hsu CM, Chen PC. Long-term outcome and efficacy of endoscopic hemorrhoid ligation for symptomatic internal hemorrhoids. World J Gastroenterol. 2011;17(19):2431-6.

14. Sun Z, Migaly J. Review of hemorrhoid disease: presentation and management. Clin Colon Rectal Surg. 2016;29(1):22-9.

15. Lin G, Ge Q, He X, Qi H, Xu L. A novel technique for the treatment of stages III to IV hemorrhoids: homemade anal cushion suspension clamp combined with harmonic scalpel. Medicine (Baltimore). 2017;96(26):e7309.

16. Akobeng AK. Understanding diagnostic tests 3: receiver operating characteristic curves. Acta Paediatr. 2007:96(5):644-7.

17. Smeenk RJ, van Lin EN, van Kollenburg P, Kunze-Busch M, Kaanders JH. Anal wall sparing effect of an endorectal balloon in 3D conformal and intensitymodulated prostate radiotherapy. Radiother Oncol. 2009;93(1):131-6.

18. Koper PC, Jansen $P$, van Putten W, van Os M, Wijnmaalen AJ, Lebesque JV, Levendag PC. Gastro-intestinal and genito-urinary morbidity after 3D conformal radiotherapy of prostate cancer: observations of a randomized trial. Radiother Oncol. 2004;73(1):1-9.

19. Paydar I, Cyr RA, Yung TM, Lei S, Collins BT, Chen LN, Suy S, Dritschilo A, Lynch JH, Collins SP. Proctitis 1 week after stereotactic body radiation therapy for prostate Cancer: implications for clinical trial design. Front Oncol. 2016:6:167.

20. Rijkmans EC, van Triest B, Nout RA, Kerkhof EM, Buijsen J, Rozema T, Franssen JH, Velema LA, Laman MS, Cats A, et al. Evaluation of clinical and endoscopic toxicity after external beam radiotherapy and endorectal 
brachytherapy in elderly patients with rectal cancer treated in the HERBERT study. Radiother Oncol. 2018;126(3):417-23.

21. Wortel RC, Witte MG, van der Heide UA, Pos FJ, Lebesque JV, van Herk M, Incrocci L, Heemsbergen WD. Dose-surface maps identifying local doseeffects for acute gastrointestinal toxicity after radiotherapy for prostate cancer. Radiother Oncol. 2015;117(3):515-20.

22. Moreau-Claeys MV, Peiffert D. Normal tissue tolerance to external beam radiation therapy: anal canalCancer Radiother. 2010;14(4-5):359-62.

\section{Publisher's Note}

Springer Nature remains neutral with regard to jurisdictional claims in published maps and institutional affiliations.

Ready to submit your research? Choose BMC and benefit from:

- fast, convenient online submission

- thorough peer review by experienced researchers in your field

- rapid publication on acceptance

- support for research data, including large and complex data types

- gold Open Access which fosters wider collaboration and increased citations

- maximum visibility for your research: over $100 \mathrm{M}$ website views per year

At $\mathrm{BMC}$, research is always in progress.

Learn more biomedcentral.com/submissions 